\title{
Cardio Protective Action of Cilostazol, Milrinone and Their Combination over Isoproterenol Induced Myocardial Infarction in Wistar Rats
}

\author{
Prashant Kumar Dhakad, ${ }^{1, *}$, Pramod Kumar Sharma ${ }^{1}$, Sokindra Kumar ${ }^{2}$, Ashish Kr. Sharma ${ }^{3}$, \\ Raghav Mishra ${ }^{1}$, Aditya Dixit ${ }^{1}$ \\ ${ }^{1}$ Department of Pharmacy, Galgotias University, India \\ ${ }^{2}$ R.V. Northland Institute (RVNI), India \\ ${ }^{3}$ Department of Pharmacology, NIMS Institute of Pharmacy, NIMS University, India
}

Copyright $\bigcirc 2017$ by authors, all rights reserved. Authors agree that this article remains permanently open access under the terms of the Creative Commons Attribution License 4.0 International License

\begin{abstract}
The cardio protective action of cilostazol, milrinone and their combination effect on myocardial infarction induced by isoproterenol subjected to Wistar rats was done by studying various parameters - heart rate, \% infarct size change, LDH level, CPKMB level, ECG pattern. In the following research work effect of synthetic drugs cilostazol, milrinone and their combined effect were studied over various biochemical and cardiac parameters. Isoproterenol was used in inducing myocardial infarction to Wistar rats. Isoproterenol $(15 \mathrm{mg} / 100 \mathrm{~g})$ administration, causes an increased levels of biochemical markers-lactate dehydrogenase $(2546.25 \pm 1.38)$, creatine phosphokinase (1485.5 .80$)$ in serum of rats indicating potential action of isoproterenol on myocardium. Due to necrosis of myocardium a marked increase of these enzymes was seen in blood. A significant decrease in heart rate (262 \pm .96$)$ in isoproterenol treated rats was observed. Infarct size in ISO group rats $(41 \% \pm .008)$ increased to certain extent with promiscuous yellow colored non-viable cells visible indicating infarction. Statistical analysis done by one-way ANOVA followed by the Dunnett's multiple comparison test where ISO control group compared with other groups, $p$ value $<0.001$ indicating extremely significant results. The present study demonstrated pharmacological benefits of combination dose of cilostazol, milrinone as evidenced by improvement in heart rate, Lactate dehydrogenase level, Creatine phosphokinase MB level, Electrocardiogram pattern, $\%$ infarct size.
\end{abstract}

Keywords Isoproterenol, Cilostazol, Milrinone, Heart Rate, Infarct Size, Lactate Dehydrogenase, Creatine Phosphokinase

\section{Introduction}

In terms of global burden of disease in 1999, the world health organization placed myocardial infarction in sixth place and stroke at seventh place, but by 2020 they will have moved to first and fourth place respectively [1]. Myocardial infarction is the irreversible damage of myocardial tissue caused by prolonged ischemia and hypoxia. This most commonly occurs when a coronary artery becomes occluded following the rupture of an atherosclerotic plaque, which then leads to the formation of a blood clot. This event can also trigger coronary vasospasm. If a vessel becomes completely occluded, the myocardium normally supplied by that vessel will become ischemic and hypoxic [2]. Without sufficient oxygen, the tissue dies. Myocardial infarctions produce clinical symptoms that include intense chest pain, substernal heaviness, squeezing, shortness of breath, fatigue, fainting, nausea, sweating, anxiety, sleeplessness, hypertension or hypotension (depending in part on the extent of cardiac damage), tachycardia and arrhythmias [3].

\subsection{Isoproterenol Induced Myocardial Infarction}

Catecholamine's administration at high doses or excess release of it from the endogenous stores may deplete the energy reserve of cardiomyocytes and thus may result in biochemical and structural changes which are responsible for the development of irreversible damage. Isoproterenol a sympathomimetic $\beta$-adrenergic receptor agonist causes severe stress to the myocardium resulting in an infarct like necrosis of the heart muscle3. The rat model of isoproterenol (ISO) induced myocardial necrosis serves as a well-accepted standardized model to evaluate several cardiac dysfunctions [4] and to study the efficacy of various natural and synthetic cardioprotective agents [5]. ISO induced myocardial 
infarction is widely used experimental model for several reasons. The model is characterized by an extraordinary technical simplicity, an excellent reproducibility as well as an acceptable low mortality [6]. Myocardial infarction induced by ISO has been reported to show many metabolic and morphological aberrations in the heart tissue of the experimental animals similar to those observed in human myocardial infarction [7]. ISO induced necrosis is maximal in the subendocardial region of the left ventricle and in the intraventricular septum [8].

Oxidative stress is, one of the main mechanisms through which catecholamines exert their toxic effects. Catecholamine-o-quinones, amino chromes and radical species resulting from the oxidation of catecholamine's are thought to be involved in catecholamine related toxicity [9]. Biochemical alterations in ISO-induced cardiomyopathy represent, a complex pattern of changes in cardiac marker enzymes, decrease in ATP store and changes in electrolyte levels in the blood as well as in the myocardial tissue [10].

Cilostazol is a phosphodiesterase inhibitor with therapeutic focus on cAMP. It inhibits platelet aggregation and is a direct arterial vasodilator. Its main effects are dilation of the arteries supplying blood to the legs and decreasing platelet coagulation. Cilostazol is a medication for the treatment of intermittent claudication [11]. Milrinone, at relevant inotropic and vasorelaxant concentrations, is a selective inhibitor of peak III cAMP phosphodiesterase isozyme in cardiac and vascular muscle. This inhibitory action is consistent with cAMP mediated increases in intracellular ionized calcium and contractile force in cardiac muscle, as well as with cAMP-dependent contractile protein phosphorylation and relaxation in vascular muscle [12].

\section{Materials and Methods}

Isoproterenol was procured from Acros Organics New Jersey, USA in the form of isoproterenol sulphate. Wistar albino rats, weighing 200-250 Gms were selected for the study. Animals were housed individually in plastic cages with filter tops under controlled conditions of 12-h light/12-h dark cycles, $50 \%$ humidity at $28^{\circ} \mathrm{C}$. The animals were allowed a standard diet and water ad libitum. They were allowed to get acclimatized in environment for one week before experimental use. The experiment was carried out as per the guidelines of the committee for the purpose of control and supervisation of experiments on animals (CPCSEA), New Delhi, India.

Standardization of Isoproterenol - Isoproterenol was initially standardized in order to limit the minimum effective concentration so as to cause myocardial infarction in rats with less or minimum mortality rate. Wistar rats were injected isoproterenol subcutaneously at dose of 15 $\mathrm{mg} / 100 \mathrm{gm}$ of body weight.

Induction of myocardial infarction- Myocardial infarction was induced in experimental rats by subcutaneously (s.c) injecting isoproterenol $(15 \mathrm{mg} / 100 \mathrm{~g}$ body weight) for 2 consecutive days.

Preparation of drug solutions- The required dose of drugs that is cilostazol, milrinone, isoproterenol were weighed accurately and dissolved in distilled water to obtain solution to be easily administered through various routes i.e. orally, intravenously, intraperitoneally, subcutaneously.

\subsection{Experimental Protocol}

After a week of acclimatization, the experimental animals were divided into 5 groups, comprising 6 rats in each group and study designed as randomized experimentation.

Group 1: Normal saline group $(\mathrm{n}=6)$

All rats receive a saline solution for about 7 days. At the end rats were anaesthetized by administering ketamine hydrochloride injection (i.p). 20 minutes after anaesthetizing rats were sacrificed and heart was excised out. Heparin solution was injected inside heart so as to clear off all blood from heart tissue.

Group 2: Isoproterenol control group $(\mathrm{n}=6)$

All rats was subjected to saline solution for 5 days and then injected with isoproterenol subcutaneously $(15 \mathrm{mg} / 100 \mathrm{~g})$ on sixth and seventh day consecutively at an interval of 24 hrs. At the end of seventh day rats were anaesthetized by administering ketamine hydrochloride (i.p), $20 \mathrm{~min}$ thereafter rats were sacrificed and their heart were excised out. Heparin solution was injected inside heart so as to clear off all blood from heart tissue.

Group 3: Cilostazol treated group $(n=6)$

All Wistar rats were initially administered the drug cilostazol $(5.8 \mathrm{mg} / \mathrm{kg})$ orally for five days and on sixth and seventh day isoproterenol $(15 \mathrm{mg} / 100 \mathrm{~g})$ was injected subcutaneously. At the end of seventh day rats were anaesthetized by administering ketamine hydrochloride (i.p), $20 \mathrm{~min}$ thereafter rats were sacrificed and their heart were excised out. Heparin solution was injected inside heart so as to clear off all blood from heart tissue.

Group4: Milrinone treated group $(\mathrm{n}=6)$

All Wistar rats were initially administered an intravenous injection of milrinone $(0.089 \mathrm{mg} / \mathrm{kg})$ for five days on lateral surface of tail vein and on sixth and seventh day isoproterenol $(15 \mathrm{mg} / 100 \mathrm{~g})$ was injected subcutaneously. At the end of seventh day rats were anaesthetized by administering ketamine hydrochloride (i.p), $20 \mathrm{~min}$ thereafter rats were sacrificed and their heart were excised out. Heparin solution was injected inside heart so as to clear off all blood from heart tissue.

Group5: Combined cilostazol and milrinone treated group $(n=6)$

All Wistar rats were subjected to a combination dose of drugs cilostazol $(5.8 \mathrm{mg} / \mathrm{kg})$ and milrinone $(0.089 \mathrm{mg} / \mathrm{kg})$ for five days and then on sixth and seventh day isoproterenol $(15 \mathrm{mg} / 100 \mathrm{~g})$ was administered (s.c) consecutively. At the end of seventh day rats were anaesthetized by administering ketamine hydrochloride (i.p), $20 \mathrm{~min}$ thereafter rats were 
sacrificed and their heart were excised out. Heparin solution was injected inside heart so as to clear off all blood from heart tissue.

At the end of seventh day all group Wistar rats were subjected to electrocardiogram apparatus for the measurement of ECG of each group rat for determining the pattern cardiac arrhythmia and heart rate in all groups. Finally at end of experimental period i.e. $24 \mathrm{hrs}$ after the last injection of ISO all group Wistar rats were sacrificed and their blood was collected for determining diagnostic biomarker enzymes- lactate dehydrogenase, creatine phosphokinase (CPK MB).

\subsection{Collection of Blood Samples}

At the end of drug treatment in each group, all the animals were fasted overnight but allowed free access to water. The next morning, blood samples were withdrawn by retro orbital puncture under mild ether anesthesia. The blood samples were collected into eppendorf tubes which were pre-coated with an anticoagulant. Blood samples were centrifuged at $3000 \mathrm{rpm}$ for $10 \mathrm{~min}$ in a refrigerated centrifuge $\left(4^{\circ} \mathrm{C}\right)$. The plasma separated as a straw colored supernatant which was used to measure various biochemical parameters. It was stored at $-20^{\circ} \mathrm{C}$ until the completion of analysis.

\subsection{Infarct Size Measurement}

After the removal of heart both of its auricles and root of aorta need to be excised and ventricles to be keep overnight at $4^{\circ} \mathrm{C}$. Freezed ventricles are to be sliced into uniform sections of 2-3 $\mathrm{mm}$ thickness. The slices then incubated in $1 \%$ triphenyl tetrazolium chloride (TTC) at $37^{\circ} \mathrm{C}$ in $0.2 \mathrm{M}$ tris buffer (PH 7.4) for $20 \mathrm{~min}$. TTC is converted into red formazone pigment by NADH and dehydrogenase enzyme and therefore stained the viable cells deep red. The infracted cells have lost the enzyme and cofactor and thus remained unstained or dull yellow. The ventricular slices are than to be placed between two glass plates. A transparent plastic grid with 100 squares in $1 \mathrm{~cm}^{2}$ is placed above it average area of each ventricular slice is calculated by counting the number squares on either side. Similarly, number of squares falling over non stained dull yellow area was also counted. Infarcted area is expressed as a percentage of total ventricular area. Whole of ventricular slices are than weighed. Infarcted dull yellow part is dissected and weighed. Infarcted size is expressed as a percentage of total ventricular area.

\subsection{Heart Rate Measurement}

Rules of 300-provided that the rhythm is regular, heart rate can be measured by dividing 300 by the number of large boxes in the R-R interval. With the ECG machine set at the standard recording speed of $25 \mathrm{~mm} / \mathrm{sec}$, the time required to record each little box on the ECG grid paper is 0.04 second. Vertically each little box is $1 \mathrm{~mm}$ in amplitude. The time required to record each large box on ECG grid paper is 0.20 second (because there are 5 little boxes in each large box, and $5 \times 0.04=0.20$ ). It can therefore be seen that the time required to record 5 large boxes will be one full second $(0.20 \times 5=1.0$ second). Thus, if a QRS complex occurs with each large box , then the R-R interval will be 0.20 second, and the rate of the rhythm is 300 beats/minute (i.e., 5 beats occur each second $\times$ 60 seconds $/$ minute $=300 /$ minute) .

$\mathrm{R}-\mathrm{R}$ interval is 2 large boxes, rate $=150$ beats $/$ minute $(300 \div 2)$

$\mathrm{R}-\mathrm{R}$ interval is 3 large boxes, rate $=100$ beats $/$ minute $(300 \div 3)$

$\mathrm{R}-\mathrm{R}$ interval is 4 large boxes, rate $=75$ beats/minute $(300 \div 4)$ and so on.

\subsection{Estimation of Lactate Dehydrogenase (LDH) and Creatine Phosphokinase}

For evaluating lactate dehydrogenase (LDH) and creatine phosphokinase, the King (1965) method was used. To standardize volumes, $0.2 \mathrm{ml} \mathrm{NAD}^{+}$solution was added to the test tubes and $0.2 \mathrm{ml}$ of water was added to control test tubes, each containing $1 \mathrm{ml}$ of the buffered substrate. The sample containing $0.01 \mathrm{ml}$ was also added to the test tubes. Test tube samples were incubated for exactly $15 \mathrm{~min}$ at $37^{\circ} \mathrm{C}$ and then arrested by adding $1 \mathrm{ml}$ of color reagent (2,4- dinitrophenyl hydrazine) to each tube and the incubation continued for an additional $15 \mathrm{~min}$. After cooling at room temperature, $10 \mathrm{ml}$ of $0.4 \mathrm{~N} \mathrm{NAOH}$ was added to each tube to make the solutions strongly alkaline. At exactly $60 \mathrm{~s}$ after the addition of alkali to each tube, the intensity of color was measured at $440 \mathrm{~nm}$.

\subsection{Statistical Analysis}

Results were expressed as mean \pm SEM. Statistical analysis of the data was done using One-way ANOVA followed by the Dunnett's multiple comparison test, where appropriate results were considered significant when $\mathrm{P}<0.05$.

\section{Results}

Standardization of isoproterenol was done in Wistar rats; it was found that the animals showed mortality chiefly at dose of $25 \mathrm{mg}$ ISO concentration administered according to body weight of Wistar rats ranging in between 200-250 Gms. The dose at which rats showed signs of myocardial infarction and without any mortality was found to be $15 \mathrm{mg}$. Myocardial infarction was determined by various biochemical parameters - lactate dehydrogenase, creatine phosphokinase, heart rate, \% infarct size in myocardium of rat heart. Normal group rats showed controlled values of all parameters- heart rate, \% infarction, LDH, CPK MB used in experimentation. Isoproterenol treated group rats showed significantly alteration in values of heart rate, $\%$ infarct size, LDH, CPK MB values, and in ECG obtained a marked ST 
wave elevation as well as QRS wave depression seen in Wistar rats. Heart rate obtained in isoproterenol treated rats showed a great decrease in its value due to the myocardial necrosis hence reduced ability of heart to contract and pump blood.

Increased ST wave elevation is a significant sign of myocardial infarction induction in rats. More precisely other parameter values were having varying difference as compared to normal group Wistar rats. Cilostazol treated rats reduces $\%$ infarct size, diagnostic marker enzymes levelslactate dehydrogenase, creatine phosphokinase to certain extent after administration of drug for sufficient period of time i.e. five days. Milrinone lactate administered group rat's responds in attenuation of the \% infarct size in myocardium, decrease in biochemical values of lactate dehydrogenase and creatine phosphokinase within serum fluid tested. Promiscuous results were seen with the combination treatment of drugs i.e. cilostazol and milrinone. There was a marked increase in heart rate due to inotropic action of drugs. \% infarction in rat heart also reduces to great extent. Enzymatic serum levels of LDH and CPK MB reduces chiefly. All these significant variations seen in parameters tested in rats against myocardial infarction suggest the therapeutically effective role of drugs in treating myocardial infarction. The recordings of electrocardiogram obtained in isoproterenol treated group rats showed an increase in ST segment elevation and decrease in QRS wave. The cilostazol, milrinone and combination of cilostazol and milrinone treated group rats electrocardiogram recordings showed the signs of improvement in heart with the outcome of nearly normal QRS and ST wave pattern. (Figure 1-6), (Table 1)

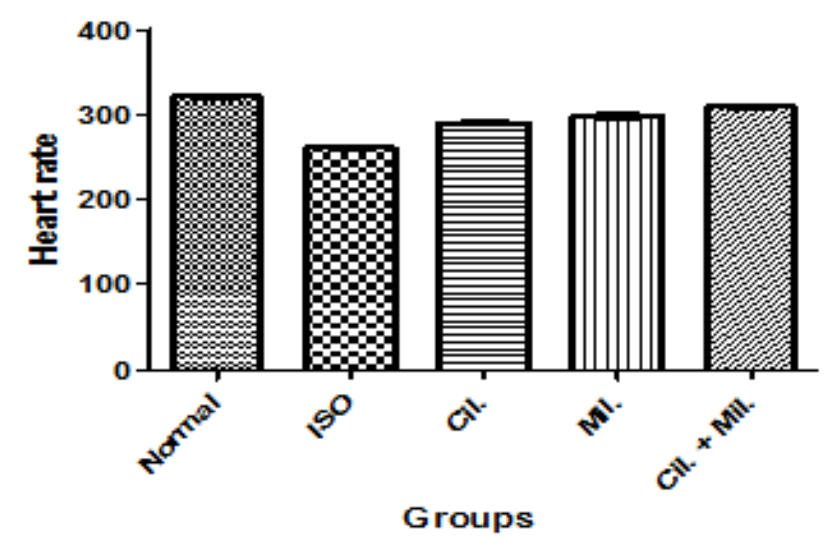

Figure 1. Values are expressed as mean \pm SEM. One-way ANOVA (non parametric test) was done among various groups followed by post dunnett's test $\&$ value obtained is extremely significant $(\mathrm{p}<0.001)$.

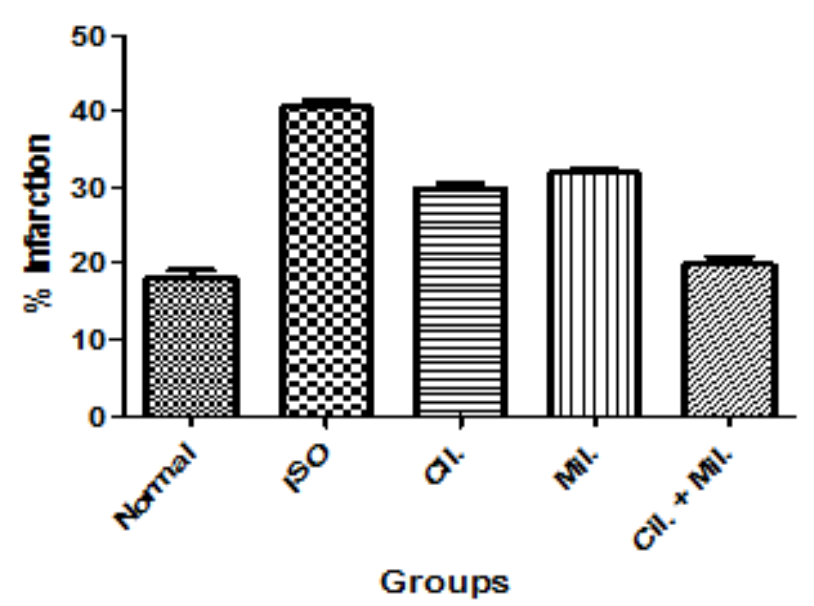

Figure 2. Values are expressed as mean \pm SEM. One-way ANOVA (non parametric test) was done among various groups followed by post dunnett's test $\&$ value obtained is extremely significant $(\mathrm{p}<0.001)$.

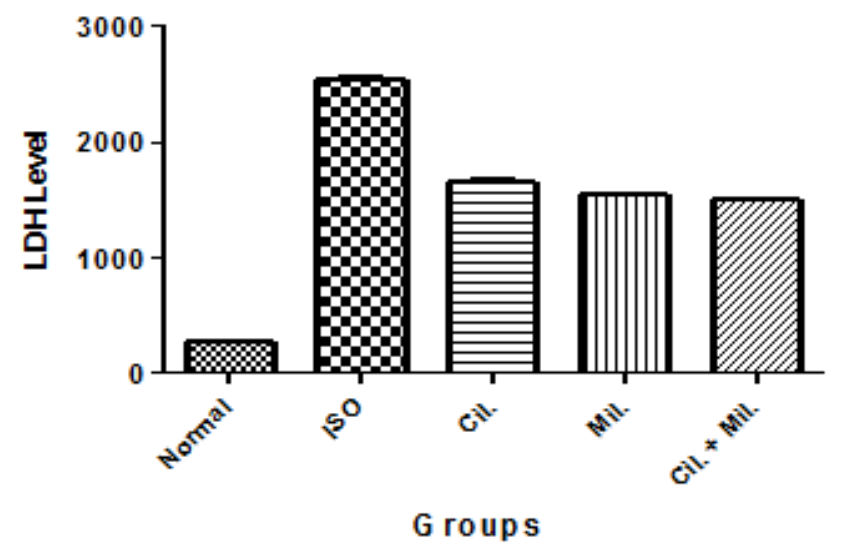

Figure 3. Values are expressed as mean \pm SEM. One-way ANOVA (non parametric test) was done among various groups followed by post dunnett's test $\&$ value obtained is extremely significant $(\mathrm{p}<0.001)$.

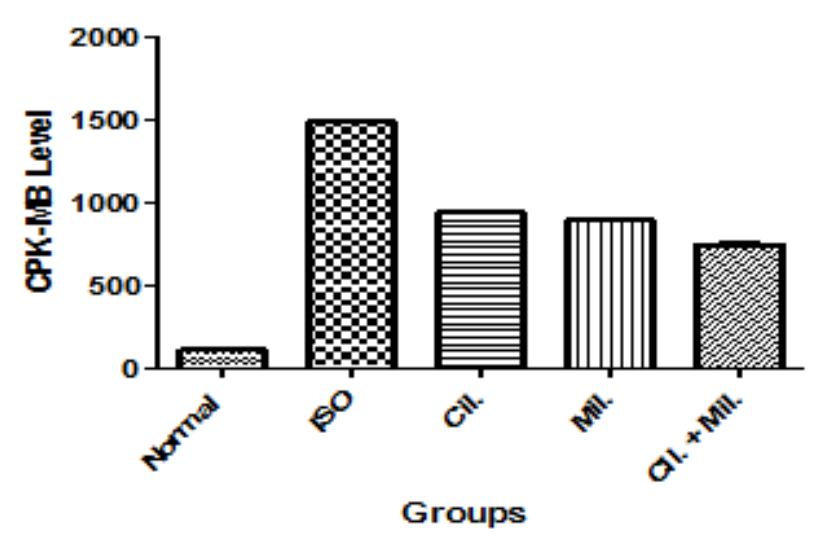

Figure 4. Values are expressed as mean \pm SEM. One-way ANOVA (non parametric test) was done among various groups followed by post dunnett 's test \& value obtained is extremely significant $(\mathrm{p}<0.001)$. 


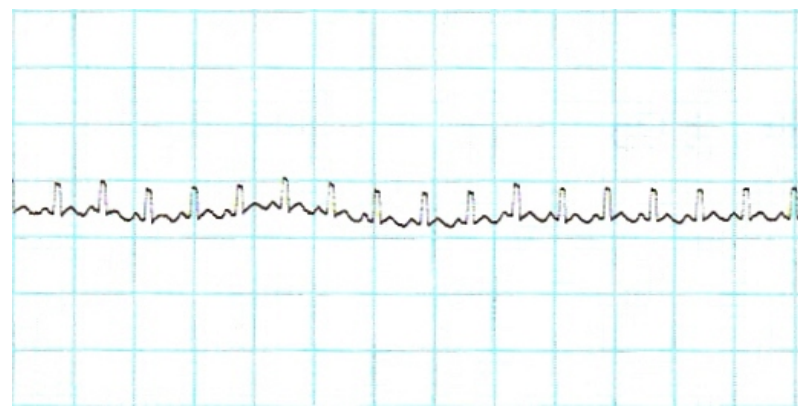

$\mathrm{A}_{1}$

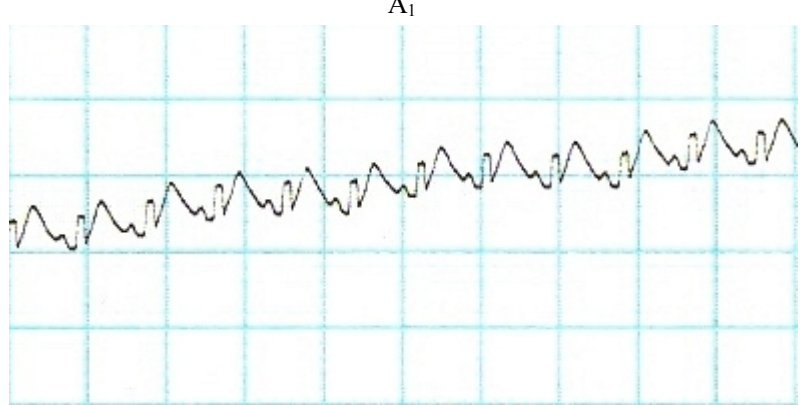

$\mathrm{B}_{1}$

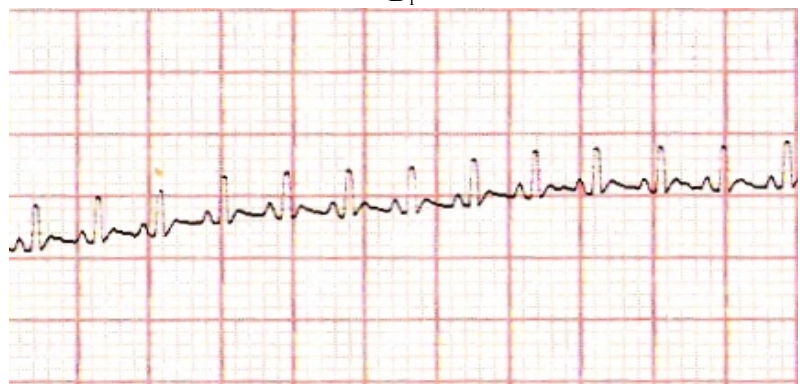

$\mathrm{C}_{1}$

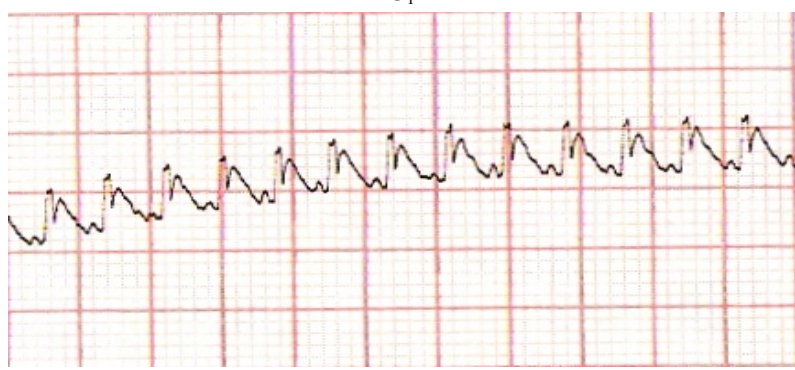

$\mathrm{D}_{1}$

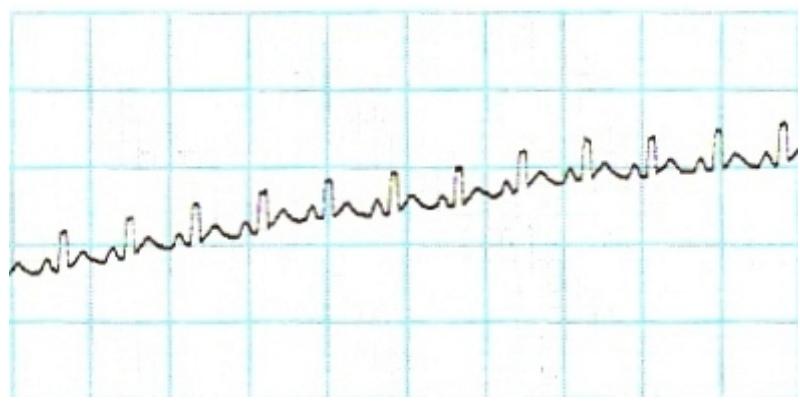

$\mathrm{E}_{1}$

Figure 5. $\mathbf{A}_{1}-\mathbf{E}_{1}$ represents $\mathrm{ECG}$ of normal saline group rats, Isoproterenol $(15 \mathrm{mg} / 100 \mathrm{~g})$ group rats, cilostazol $(5.8 \mathrm{mg} / \mathrm{kg})$ treated rats, milrinone $(0.089 \mathrm{mg} / \mathrm{kg})$ treated rats and combination of cilostazol $(5.8 \mathrm{mg} / \mathrm{kg})$ and milrinone $(0.089 \mathrm{mg} / \mathrm{kg})$ treated rats obtained $24 \mathrm{hrs}$ after the last dose of ISO respectively.

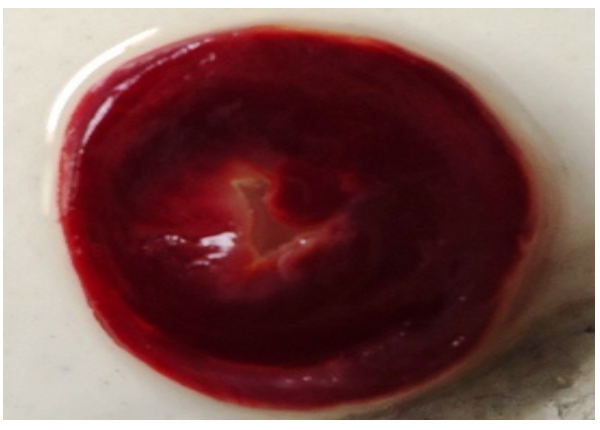

$\mathrm{A}_{2}$

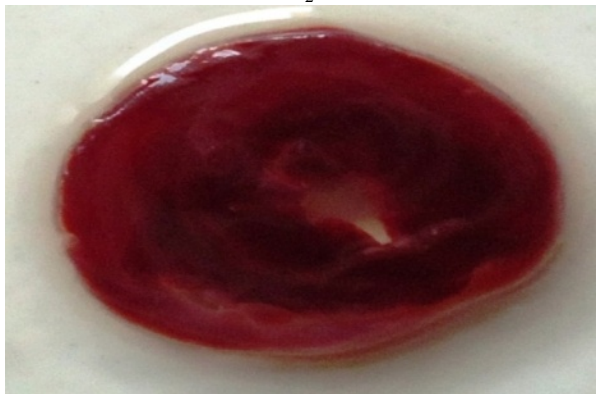

$\mathrm{B}_{2}$

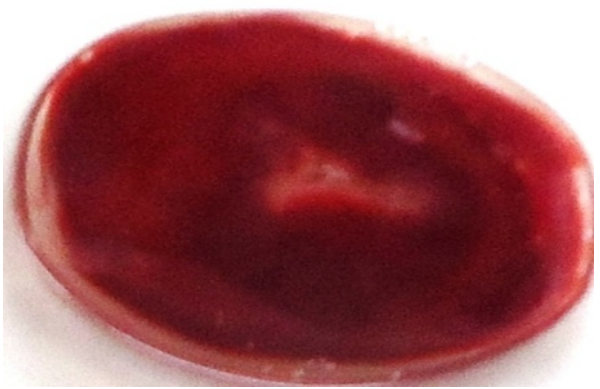

$\mathrm{C}_{2}$

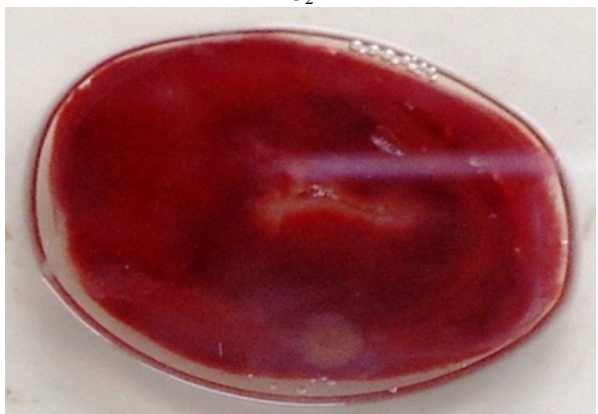

$\mathrm{D}_{2}$

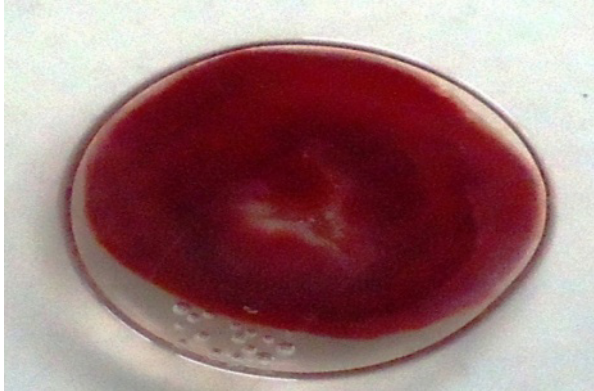

$\mathrm{E}_{2}$

Figure 6. $\quad \mathbf{A}_{2}-\mathbf{E}_{2}$ represents myocardium of normal saline group treated, isoproterenol treated group Wistar rats showing infarction (yellow coloured non-viable cells), cilostazol treated Wistar rats, milrinone treated Wistar rats and combination of cilostazol and milrinone treated group Wistar rats 
Table 1. Observation table consisting of various parameters expressing the experimentally obtained values within different groups of Wistar rats

\begin{tabular}{|c|c|c|c|c|c|}
\hline S. No & Groups & Heart Rate & $\%$ Infarct Size & LDH level & CPK MB level \\
\hline 1 & Normal saline & $321 \pm .94$ & $18 \% \pm .009$ & $255 \pm 1.29$ & $103.66 \pm .88$ \\
\hline 2 & Isoproterenol control & $262 \pm .96 * * *$ & $41 \% \pm .008 * * *$ & $2546.25 \pm 1.38^{* * *}$ & $1485.5 \pm .80^{* * *}$ \\
\hline 3 & Cilostazol treated & $291 \pm .84 * \mathrm{a}$ & $30 \% \pm .007 * \mathrm{a}$ & $1659.5 \pm 0.99 * \mathrm{a}$ & $932.5 \pm .75 * \mathrm{a}$ \\
\hline 4 & Milrinone treated & $301 \pm .1 .16 * \mathrm{a}$ & $31.83 \% \pm .006 * \mathrm{a}$ & $1530.58 \pm 1.44 * \mathrm{a}$ & $893.5 \pm .75 * \mathrm{a}$ \\
\hline 5 & Cilostazol + milrinone treated & $310 \pm .76 * \mathrm{a}$ & $20 \% \pm .010 * \mathrm{a}$ & $1501.25 \pm 1.19 * \mathrm{a}$ & $742.9 \pm .93 * \mathrm{a}$ \\
\hline
\end{tabular}

Values are expressed as mean $\pm \operatorname{SEM}(\mathrm{n}=6) .{ }^{* * *} \mathrm{p}<0.001$ ISO control vs all other groups in dunnett's multiple comparison test.

\section{Discussions}

In the present study, isoproterenol-induced myocardial injury has been shown to produce a significant cardiac dysfunction in consonance to previous studies as evidenced by decreased HR (heart rate) after isoproterenol administration. Cilostazol and milrinone pretreatment significantly prevented the decrease in HR (determinants of myocardial oxygen demand), thereby decreased workload and facilitated the heart to maintain myocardial oxygen balance in ischemic tissues. Restoration of altered HR also increased blood flow through the subendocardial region, which bears the maximal burnt of ischemic insult in isoproterenol- induced myocardial infarction. These beneficial effects were translated into functional recovery of the heart under ischemic conditions.

One-way ANOVA was done and followed by Dunnett's test where ISO control group was compared to a rest other groups and a significant result was obtained.

After the five days pretreatment of drugs cilostazol, milrinone, and combination of cilostazol and milrinone to Wistar rats, isoproterenol was injected (s.c) and the effect of these drugs were found out over various parameters. In this experiment we have initially compared the values of different parameters obtained in normal saline treated rats and isoproterenol treated rats. Thereafter the drug treated group rats values were compared with the isoproterenol group treated rats and normal group treated rats. The various parameters were - heart rate, percent infarct size measurement, lactate dehydrogenase levels, creatine phosphokinase levels, changes in electrocardiogram pattern of Wistar rats.

Heart rate in normal saline treated rats is observed as $321 \pm .94$ while isoproterenol treated rats showed a significant decrease in value i.e. $262 \pm .96$. Possible reasons for this decrement may be due to necrosis of myocardial cells whose capacity reduces with span of time due to infarction. On the other hand cilostazol treated rats group showed an improvement in heart rate with value as $291 \pm .84$, milrinone treated rats group showed heart rate as $301 \pm 1.16$. It is noted that milrinone is more effective than cilostazol in returning the heart rate to the normal range value. Combination of cilostazol and milrinone when administered to rats showed an additive effect to heart rate as the value obtained is $310 \pm .76$ which corresponds to the effective role of combination therapy of cilostazol and milrinone in myocardial infarction since achieving to normal heart rate. Percent infarct size in rats with myocardial infarction signifies the percentage of viable cells and that of non-viable cells present in heart muscles. \% myocardial infarction in normal saline treated rats is $18 \% \pm .009$ while in isoproterenol treated rats were $41 \% \pm .008$ which is marked increase in number of infarcted cells. Cilostazol treated rats group showed \% infarction as $30 \% \pm .007$ which certainly brought down the higher value of $41 \%$ to $30 \%$ of infarction signifying effectiveness of drug. Milrinone treated rats group showed a value of $31 \% \pm .006$. Combination of cilostazol and milrinone treated rats group showed a value of $20 \% \pm .01$ as $\%$ infarction. It is seen after assessing the values obtained of \% infarction in various group of Wistar rats, that combination therapy is much more effective in treating the myocardial infarction as compared to the individual therapy of cilostazol and milrinone.

Another parameter is lactate dehydrogenase enzyme in heart. Marked increase in total LDH occurs in myocardial infarction. LDH-1 is found mainly in the heart, an LDH-1 level higher than that of LDH-2 is indicative of a heart attack or injury. Normally, the level of LDH-2 is higher than the level of LDH-1 in serum. When LDH-1 level becomes higher than that of LDH-2, a phenomenon known as "flipped $\mathrm{LDH} "$ is seen which is a strong indicator of a heart attack (damage to heart muscles releases LDH, which is rich in LDH 1 in bloodstream). Normal saline group treated rats obtained an LDH level value as nearly normal $255 \pm 1.29$ IU/L. Isoproterenol treated group rats showed an abrupt increase in lactate dehydrogenase level as $2546.25 \pm 1.38$ $\mathrm{IU} / \mathrm{L}$ compared to normal saline rats. It is because of myocardial infarction in heart that reduces LDH in myocytes and tends it to secrete in serum leading to increased serum LDH levels. Cilostazol treated rats showed LDH value as $1659.5 \pm .99 \mathrm{IU} / \mathrm{L}$ while milrinone treated rats showed value of $1530.58 \pm 1.44 \mathrm{IU} / \mathrm{L}$.Combination of cilostazol and milrinone treated rats reduces the lactate dehydrogenase levels to much extent with value as $1501.25 \pm 1.19 \mathrm{IU} / \mathrm{L}$. The results of combination therapy of drugs decrease the blood LDH levels in rats with infarction which signify the cardio protective its effect.

CPK-MB appears to be the most sensitive and most specific indicator of the acute myocardial infarction. There are three isoenzymes of $\mathrm{CPK}-\mathrm{MM}, \mathrm{MB}$, and $\mathrm{BB}$. CPK-MB is present utmost within heart muscles. Biomarker enzyme creatine phosphokinase (CPK MB) level found in the normal saline treated rats was $103.66 \pm .88$. CPK level found in isoproterenol treated rats group is $1485.5 \pm .80$; well it seems to be much more than compared to the normal saline treated rats. The level of creatine phosphokinase rises in case of 
myocardial infarcted heart due to necrosis in myocardium. Level of CPK-MB found in cilostazol treated rats is $932.5 \pm .75$ which is positive indication in treating infarction. Milrinone treated rats group showed CPK level as $893.5 \pm .75$, it can be seen that milrinone reduces the level of creatine phosphokinase in serum to greater extent than cilostazol. Level CPK-MB reduces to a marked extent in rats treated with combination therapy of cilostazol and milrinone with value obtained as $742.9 \pm .93$. It can be concluded from the results obtained that combination therapy reduces the CPK-MB levels more as compared to individual therapies of cilostazol and milrinone.

The most prominent feature of marked recognition in myocardial infarction within heart of Wistar rats is changes in electrocardiogram. ECG of normal saline group rats is absolutely within normal range. Electrocardiogram of isoproterenol treated group rats produce a significant change i.e. elevation of ST wave and depression of QRS complex. Cilostazol and Milrinone treated group rats produces ECG as close to normal group rats. Combination therapy produces decrease in ST wave segment and rise in QRS complex tending it to be normal and hence showing cardio protective role against myocardial infarction.

\section{Conclusions}

The present study findings demonstrated therapeutic benefits of cilostazol, milrinonein an integrated approach as evidenced by restoration of hemodynamic and contractile function, improvement in endogenous antioxidant defense, Heart rate, lactate dehydrogenase, creatinine phosphokinase levels, electrocardiogram changes. Biomarker enzymes lactate dehydrogenase, creatine phosphokinase levels decreases to a certain extent after drug therapy treatment. The study has also revealed that combination therapy of cilostazol and milrinone is an effective modality that could alleviate symptoms and might be used for prophylaxis and treatment of myocardial infarction. The effectiveness of drug for cardiovascular disease is believed to be mediated by its complete composition and interactions, which can target many signal transduction and metabolic pathways and consequently, reduce cardiovascular symptoms.

Cardiac arrthymia pattern is observed in isoproterenol induced Wistar rats. Cilostazol treated rats showed positive signs in rhythm of heart similarly milrinone improve the cardiac rhythm. Combination effect of cilostazol and milrinone shows miraculous results by returning the normal heart rhythm pattern.

Heart rate of isoproterenol treated rat's decreases initially that normalizes after treatment with cilostazol and milrinone while when injected with combination drug treatment showed significant results by increment of heart rate to the normal range. This may be due to positive inotropic and lusinotropic effects of cilostazol and milrinone.
Myocardial infarct size pattern observed in all five groups indicates the reduction in percent infarction of myocardium of cilostazol and milrinone treated rat hearts as compared to increased infarct size in isoproterenol treated Wistar rats.

Finally after studying the therapeutic role, assessing the prior studies on treatment therapies of myocardial infarction and values of various parameters- heart rate, lactate dehydrogenase level, creatine phosphokinase level, \% infarction, ECG pattern of cilostazol and milrinone on isoproterenol induced myocardial infarction it may be concluded that although individual therapies of drug cilostazol and milrinone is effective but the combination therapy of cilostazol and milrinone produces remarkably significant results in treatment against myocardial infarction.

\section{Conflict of Interest}

Authors declare that there are no conflicts of interest.

\section{REFERENCES}

[1] WHO. World Health Organization in collaboration with the Centre for Diseases Control and Prevention, Geneva, Switzerland. 2004; pp.48-49

[2] Sardha A, Jhan TMG. Cardiovascular disease treatment strategies. Health Action 2009; 22:28-31.

[3] Sushma KS, Jaydeep A, Kumar JSS. Effect of carnitine on malondialdehyde, taurine and glutathione levels in heart of rats subjected to myocardial stress by isoproterenol. Ind. J. Exp. Ther. 1989; 214:688-693.

[4] Wexler BC. Myocardial infarction in young vs old male rats: Pathophysiological changes. Am. Heart J. 1978; 96: 70-78.

[5] Rathore N, John S, Kale M. Lipid peroxidation and antioxidant enzymes in isoproterenol induced oxidative stress in rat tissues. Pharmacol. Res. 1988; 38: 297-303.

[6] Grimm D, Elsner D, Schunkert H. Development of heart failure following isoproterenol administration in the rat: Role of rennin angiotensin system. Cardiovas Res. 1988; 37: 91-100.

[7] Nirmala C, Puvanakrishnan R. Influence of curcumin treatment on collagen metabolism in experimental myocardial necrosis in rats. Mol. Cell. Biochem. 1999; 111: 231-237.

[8] Bolutyl MO, Long X, Exchanhagen T. Isoproterenol infusion alterations in expression of hypertrophy-associated genes in rat heart. Am. J. Physiol. 1995; 269: H638-H647.

[9] Rupp H, Dhalla KS, Dhalla NSJ. Cardivasc. Pharmacol. 1994; 24:16-16.

[10] Lehr D. Healing of myocardial necrosis caused by sympathomimetic amines. Recent. Adv. Stud. Cardiac. Struct. Metlab. 1972; 1:526-550.

[11] http://www.drugs.com/pro/cilostazol.html

[12] http://www.drugs.com/pro/milrinone.html 